\title{
First-principles investigation of magnetic and transport properties in hole-doped shandite compounds $\mathrm{Co}_{3} \mathrm{In}_{x} \mathrm{Sn}_{2-x} \mathrm{~S}_{2}$
}

\author{
Yuki Yanagi, ${ }^{1}$ Junya Ikeda, ${ }^{2}$ Kohei Fujiwara $\odot,{ }^{2}$ Kentaro Nomura, ${ }^{2,3}$ Atsushi Tsukazaki, ${ }^{2,3,4}$ and Michi-To Suzuki $\odot^{1,5}$ \\ ${ }^{1}$ Center for Computational Materials Science, Institute for Materials Research, Tohoku University, Sendai, Miyagi 950-8577, Japan \\ ${ }^{2}$ Institute for Materials Research, Tohoku University, Sendai Miyagi 950-8577, Japan \\ ${ }^{3}$ Center for Spintronics Research Network, Tohoku University, Sendai, Miyagi 980-8577, Japan \\ ${ }^{4}$ Center for Science and Innovation in Spintronics (CSIS), Core Research Cluster, Tohoku University, Sendai, Miyagi 980-8577, Japan \\ ${ }^{5}$ Center for Spintronics Research Network, Graduate School of Engineering Science, Osaka University, Toyonaka, Osaka 560-8531, Japan
}

(Received 30 November 2020; revised 31 March 2021; accepted 14 April 2021; published 7 May 2021)

\begin{abstract}
Co-based shandite $\mathrm{Co}_{3} \mathrm{Sn}_{2} \mathrm{~S}_{2}$ is a representative example of magnetic Weyl semimetals showing rich transport phenomena. We thoroughly investigate magnetic and transport properties of hole-doped shandites $\operatorname{Co}_{3} \operatorname{In}_{x} \operatorname{Sn}_{2-x} \mathrm{~S}_{2}$ by first-principles calculations. The calculations reproduce nonlinear reduction of anomalous Hall conductivity with doping In for $\mathrm{Co}_{3} \mathrm{Sn}_{2} \mathrm{~S}_{2}$ as reported in experiments against the linearly decreased ferromagnetic moment within virtual crystal approximation. We show that a drastic change in the band parity character of Fermi surfaces, attributed to the nodal rings lifted energetically with In doping, leads to strong enhancement of anomalous Nernst conductivity with reversing its sign in $\mathrm{Co}_{3} \mathrm{In}_{x} \mathrm{Sn}_{2-x} \mathrm{~S}_{2}$.
\end{abstract}

DOI: 10.1103/PhysRevB.103.205112

\section{INTRODUCTION}

Novel charge and spin transports have attracted growing interests in the contexts of topological phases of matter. The anomalous Hall effect (AHE) and the anomalous Nernst effect (ANE) are classical examples of such phenomena and have been studied for many years [1]. The AHE (ANE) is characterized by a transverse charge current flow $\boldsymbol{j}$ induced by an applied electric-field $\boldsymbol{E}$ (thermal gradient $\nabla T$ ) in the absence of an external magnetic field as follows:

$$
j_{a}=\sum_{b}\left[\sigma_{a b}^{\mathrm{A}} E_{b}+\alpha_{a b}^{\mathrm{A}}\left(-\nabla_{b} T\right)\right],
$$

where antisymmetric tensors $\sigma_{a b}^{\mathrm{A}}$ and $\alpha_{a b}^{\mathrm{A}}$ are the anomalous Hall conductivity (AHC) and the anomalous Nernst conductivity (ANC), respectively, with $a, b=x, y, z$. Since the $\mathrm{AHC}$ and ANC are closely related to topological properties of electronic structures via the Berry curvature [1-5], significant effort has been devoted to exploring such anomalous transports in topological quantum matters with time-reversal symmetry breaking. Recently, large anomalous Hall and Nernst responses have been observed in magnetic Weyl semimetals including $\mathrm{Co}_{3} \mathrm{Sn}_{2} \mathrm{~S}_{2}$ and $\mathrm{Co}_{2} \mathrm{MnGa}$ [6-10].

Shandite compound $\mathrm{Co}_{3} \mathrm{Sn}_{2} \mathrm{~S}_{2}$ is a half-metallic ferromagnet with transition temperature $T_{\mathrm{c}} \sim 177 \mathrm{~K}$ and saturated moment $M \sim 0.3 \mu_{\mathrm{B}}$ per Co atom [11-15]. This material is a representative example of magnetic Weyl semimetals since,

Published by the American Physical Society under the terms of the Creative Commons Attribution 4.0 International license. Further distribution of this work must maintain attribution to the author(s) and the published article's title, journal citation, and DOI. according to the angle-resolved photoemission spectroscopy (ARPES) and electronic structure calculations, Weyl nodes are located near the Fermi energy $[6,7,16]$. In Weyl semimetals, the divergent behavior of the Berry curvature at the Weyl nodes can give rise to characteristic physics, e.g., the chiral magnetic effect and the emergence of the anomalous surface states called Fermi arcs [3]. The existence of the anomalous surface states in $\mathrm{Co}_{3} \mathrm{Sn}_{2} \mathrm{~S}_{2}$ has been confirmed by the ARPES and scanning tunneling spectroscopy/microscopy (STS/M) measurements [16-18], supporting the existence of the Weyl nodes near the Fermi energy. In addition, importance of the Berry phase effects in low-energy physics has been indicated by the STS/M measurements [19,20].

$\mathrm{Co}_{3} \mathrm{Sn}_{2} \mathrm{~S}_{2}$ is expected to be a potential candidate for the thermoelectric devise applications due to the characteristic transport phenomena with the large AHC and ANC, reaching $\sigma_{x y}^{\mathrm{A}} \sim 500-1130 \mathrm{~S} \mathrm{~cm}^{-1}[6,7,14,15]$ and $\alpha_{x y}^{\mathrm{A}} \sim$ $2-10 \mathrm{~A} \mathrm{~K}^{-1} \mathrm{~m}^{-1}[8,21]$, respectively. Previous theoretical studies imply the close relation between these anomalous transports and topological bands, such as Weyl nodes and nodal rings [1-3,5-7,22-24]. Controlling pressure, temperature, and chemical composition often affect transport properties. It is known that, in $\mathrm{Co}_{3} \mathrm{Sn}_{2} \mathrm{~S}_{2}$, applying pressure suppresses the AHE, leading to the AHC and Hall angle comparable to those in conventional ferromagnetic metals [25-27].

It has also been shown experimentally that the chemical substitution has a considerable impact on the magnetic and transport properties. So far, the effects of $\mathrm{Fe}$ or Ni substitution for Co and In substitution for Sn have been investigated [11,28-32] where Ni substitution corresponds to the electron doping, whereas $\mathrm{Fe}$ and In substitutions correspond to the hole doping. Irrespective of doped elements, similar doping dependence of magnetic properties has been confirmed, that 


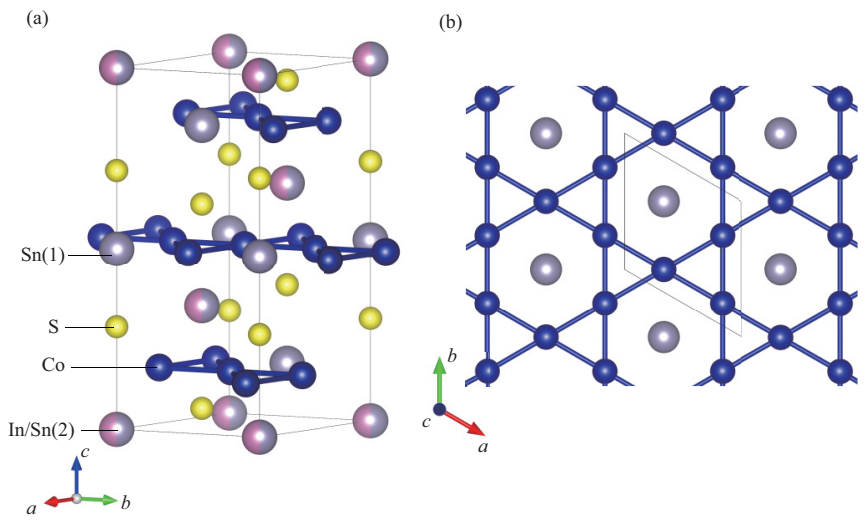

FIG. 1. (a) Crystal structure of $\mathrm{Co}_{3} \mathrm{In}_{x} \mathrm{Sn}_{2-x} \mathrm{~S}_{2}$, and (b) that on the Co-kagome layer. The solid line represents the conventional unit cell which is three times larger than the primitive one. These figures are drawn by using VESTA [34].

is, the monotonic decrease in the transition temperature and magnetic moment with increasing doping content. The doping effects on transport properties, on the other hand, show more complicated behaviors. In the case of the $\mathrm{Ni}$ substitution, the AHC decreases monotonically with the doping content [31], whereas in the cases of Fe and In substitutions, AHC is enhanced for relatively small doping and decreases for large doping [30,32]. From the theoretical side, the effects of substitution atoms on magnetic properties and/or electric transport properties have been previously investigated based on the first-principles calculations with the supercell approach for specific doping concentrations [29-31,33]. A systematic investigation of the atom substitution effects on the thermoelectric transport, i.e., the Nernst effect has not been performed neither experimentally and theoretically. Here, we present a systematic investigation of the effects of In doping into $\mathrm{Sn}$ sites on the thermoelectric transports as well as magnetic properties based on the first-principles method.

\section{METHODS}

We perform the density functional calculation for $\mathrm{Co}_{3} \mathrm{In}_{x} \mathrm{Sn}_{2-x} \mathrm{~S}_{2}$ by using the WIEN2K code $[35,36]$ where the generalized gradient approximation (GGA) by Perdew, Burke, and Ernzerhof is employed for the exchange-correlation functional [37]. Note that GGA is a good approximation for itinerant magnets [38] and actually works well for describing magnetism in $\mathrm{Co}_{3} \mathrm{Sn}_{2} \mathrm{~S}_{2}$ [6,7]. Muffin-tin radii for $\mathrm{Co}, \mathrm{In} / \mathrm{Sn}$, and $\mathrm{S}$ atoms are set to $2.26,2.5$, and 1.85 a.u., respectively, and plane-wave cutoff $K_{\max }$ is set so as to satisfy $R_{\mathrm{MT}} K_{\max }=8$ where a.u. represents the Bohr radius, and $R_{\mathrm{MT}}$ is the smallest muffin-tin radius. We sample the Brillouin zone with $22^{3} \boldsymbol{k}$ points for the self-consistent electronic structure calculation.

$\mathrm{Sn} /$ In substitution effects are treated within the virtual crystal approximation (VCA) and with the lattice parameters adopted from the experimental values of $\mathrm{Co}_{3} \mathrm{Sn}_{2} \mathrm{~S}_{2}$ in Ref. [29], independent of In content $x$. Sn atoms in $\mathrm{Co}_{3} \mathrm{Sn}_{2} \mathrm{~S}_{2}$ occupy two inequivalent Wyckoff positions $\operatorname{Sn}(1)$ and $\operatorname{Sn}(2)$ sites for intra- and inter-Co kagome layer sites, respectively, as shown in Fig. 1. In this work, $\mathrm{Sn}$ atoms at the $\mathrm{Sn}(2)$ site are substituted by In atoms for the proper description of the nonmagnetic insulating phase at $x=1.0$ [33]. In the VCA, the system at the In-content $x$ is modeled by the fictitious atom at the $\operatorname{Sn}(2)$ site having effective atomic number $Z_{\text {eff }}=$ $(1-x) Z_{\text {Sn }}+x Z_{\text {In }}$ with the number of valence electrons decreased by $x$, where $Z_{\mathrm{Sn}}=50$ and $Z_{\text {In }}=49$ are the atomic numbers for $\mathrm{Sn}$ and In atoms, respectively.

We generate the maximally localized Wannier function to construct an effective tight-binding Hamiltonian from the obtained electronic structures with WANNIER90 package $[39,40]$ through the WIEN2WANNIER interface [41], which enables us accurately calculate the transport properties with an acceptable numerical cost [42]. We explicitly include 58 Wannier orbitals, i.e., Co- $3 d, \mathrm{Sn} / \mathrm{In}-5 s, 5 p$, and S-3p orbitals for the Wannier model to assure high reproducibility of the electronic structures obtained by density functional theory calculations. These orbitals are derived from 62 target Bloch states around the Fermi energy. When the number of Bloch states is larger than that of Wannier orbitals, we have to perform the disentanglement procedure to construct an optimal subspace within the outer energy window. In the present calculation, lower (upper) bound $E_{\min }\left(E_{\max }\right)$ of the outer energy window is set to the bottom (top) of the lowest (highest) target energy bands. For example, we set $E_{\min }=-9.39$ and $E_{\max }=6.71 \mathrm{eV}$ for $\mathrm{Co}_{3} \mathrm{Sn}_{2} \mathrm{~S}_{2}$ and set $E_{\min }=-8.99$ and $E_{\max }=7.23 \mathrm{eV}$ for $\mathrm{Co}_{3} \mathrm{InSnS}{ }_{2}$ where the energy is measured from the Fermi level. In addition to the outer energy window, we can set the inner energy window in which the electronic states of Wannier models completely reproduce those obtained by firstprinciples calculations. The inner energy window is set to $\left[E_{\min }, 2.0 \mathrm{eV}\right]$ in this work.

Based on the Wannier models, we investigate the intrinsic contributions to electric and thermoelectric transports, which are determined from purely electronic band structures in $\mathrm{Co}_{3} \mathrm{In}_{x} \mathrm{Sn}_{2-x} \mathrm{~S}_{2}$ by calculating the AHC and ANC with use of the following Kubo formulas [1,42-44],

$$
\begin{aligned}
& \sigma_{a b}^{\mathrm{A}}(\mu, T)=-\frac{e^{2}}{\hbar} \int_{\mathrm{BZ}} \frac{d \boldsymbol{k}}{(2 \pi)^{3}} \sum_{n} f\left(\varepsilon_{n \boldsymbol{k}}\right) \Omega_{n, a b}(\boldsymbol{k}), \\
& \alpha_{a b}^{\mathrm{A}}(\mu, T)=\frac{e k_{\mathrm{B}}}{\hbar} \int_{\mathrm{BZ}} \frac{d \boldsymbol{k}}{(2 \pi)^{3}} \sum_{n} s\left(\varepsilon_{n \boldsymbol{k}}\right) \Omega_{n, a b}(\boldsymbol{k}),
\end{aligned}
$$

where $e, k_{\mathrm{B}}, \hbar$, and $\varepsilon_{n k}$ are the positive elementary charge, Boltzmann constant, reduced Planck constant, one-particle energy with band index $n$, and wave-vector $\boldsymbol{k}$, respectively. The Fermi distribution function $f(\varepsilon)$ and the entropy density $s(\varepsilon)$ are given as $f(\varepsilon)=\left(e^{(\varepsilon-\mu) / k_{\mathrm{B}} T}+1\right)^{-1}$ and $s(\varepsilon)=-f(\varepsilon) \ln f(\varepsilon)-[1-f(\varepsilon)] \ln [1-f(\varepsilon)] . \Omega_{n, a b}(\boldsymbol{k})$ is the Berry curvature for band $n$, which is expressed as follows:

$$
\Omega_{n, a b}(\boldsymbol{k})=-2 \hbar^{2} \operatorname{Im} \sum_{m(\neq n)} \frac{\left\langle n \boldsymbol{k}\left|v_{a}\right| m \boldsymbol{k}\right\rangle\left\langle m \boldsymbol{k}\left|v_{b}\right| n \boldsymbol{k}\right\rangle}{\left(\varepsilon_{n \boldsymbol{k}}-\varepsilon_{m \boldsymbol{k}}\right)^{2}}
$$

where $v_{a}$ is the velocity operator along the $a$ direction, $|n \boldsymbol{k}\rangle$ is the Bloch state with band index $n$, and wave-vector $\boldsymbol{k}$. In the actual numerical calculation, we perform the $\boldsymbol{k}$ integration in Eqs. (2) and (3) as the discrete $\boldsymbol{k}$ summation on $250^{3}$ grids in the first Brillouin zone. 

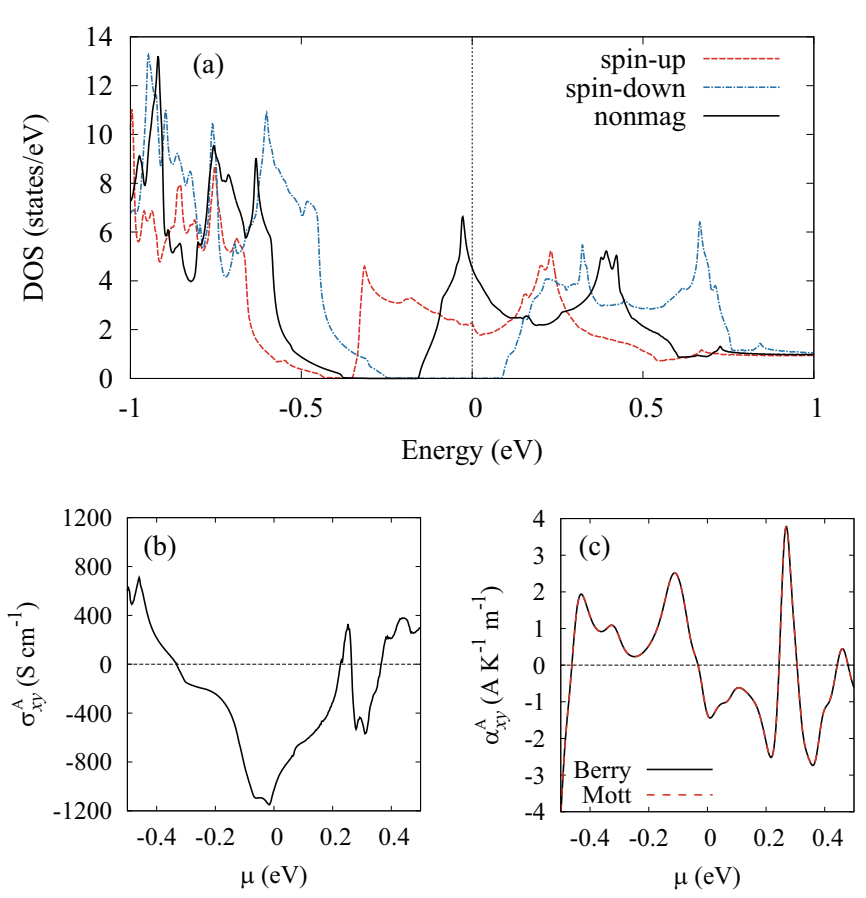

FIG. 2. (a) DOS for $\mathrm{Co}_{3} \mathrm{Sn}_{2} \mathrm{~S}_{2}$ in nonmagnetic and ferromagnetic states. The solid and dashed (dot-dashed) lines represent DOS per spin in the nonmagnetic state and that with spin-up (-down) in the ferromagnetic state, respectively. The chemical potential dependences of (b) AHC at $k_{\mathrm{B}} T=0$ and (c) ANC at $k_{\mathrm{B}} T=0.01 \mathrm{eV}$. In panel (c), the solid and dashed lines represent the ANCs calculated via the Berry phase formula in Eq. (3) and generalized Mott formula in Eq. (5), respectively.

\section{RESULTS}

First, we show the results for $x=0$, i.e., $\mathrm{Co}_{3} \mathrm{Sn}_{2} \mathrm{~S}_{2}$. Figure 2(a) shows the density of states (DOS) for $\mathrm{Co}_{3} \mathrm{Sn}_{2} \mathrm{~S}_{2}$ both in the nonmagnetic and in the ferromagnetic states. The DOS in the nonmagnetic state has a gap between -0.31 to $-0.17 \mathrm{eV}$ and the states from $-0.17 \mathrm{eV}$ to the Fermi energy $E_{\mathrm{F}}$ are occupied by one electron per unit cell, resulting in the fully polarized magnetic moment $\sim 1 \mu_{\mathrm{B}}$ per unit cell in the ferromagnetic calculation. Since the primitive unit cell contains three Co atoms, this corresponds to $\sim 0.33 \mu_{\mathrm{B}}$ per Co atom and is consistent with the experimental value of $\sim 0.3 \mu_{\mathrm{B}}$ per Co atom from magnetization measurements [13,14]. We here briefly describe valence and spin states of each ion. In the present Wannier model, the numbers of occupied electrons at $\mathrm{Co}, \operatorname{Sn}(1), \operatorname{Sn}(2)$, and $\mathrm{S}$ ions are 8.02, 4.72, 4.69, and 4.76 , respectively, in the nonmagnetic state. The $84 \%$ of one-electron states from $-0.17 \mathrm{eV}$ to $E_{\mathrm{F}}$ in the nonmagnetic calculation are composed of the $d$ orbitals from Co ions. In the ferromagnetic state, magnetization on a Co atom is $0.352 \mu_{\mathrm{B}}$, which is slightly larger than $0.33 \mu_{\mathrm{B}}$ and the magnetizations on $\mathrm{Sn}(1), \mathrm{Sn}(2)$, and $\mathrm{S}$ sites are $-0.051,-0.049$, and $0.022 \mu_{\mathrm{B}}$, respectively. The exchange splitting pushes down the up-spin DOS, leading to the Fermi energy around the dip of the upspin DOS, and pushes up the down-spin DOS, resulting the Fermi energy in a gap of down-spin DOS. As a consequence, the half-metallic state is realized.
The electric and thermoelectric transport properties in $\mathrm{Co}_{3} \mathrm{Sn}_{2} \mathrm{~S}_{2}$ are now discussed. Since the Berry curvature and ferromagnetic moment have the same symmetry properties under the magnetic point group, the AHC and ANC can be finite in the ferromagnetic state [42,46-50]. In the present case since the magnetic point group of the ferromagnetic phase is $\overline{3} m^{\prime}$, only $\sigma_{x y}^{\mathrm{A}}=-\sigma_{y x}^{\mathrm{A}}$ and $\alpha_{x y}^{\mathrm{A}}=-\alpha_{y x}^{\mathrm{A}}$ can be finite, and the other components vanish. It is instructive to rewrite ANC in Eq. (3) by the generalized Mott formula as follows [44]:

$$
\begin{gathered}
\alpha_{a b}^{\mathrm{A}}(\mu, T)=\frac{1}{e T} \int d \varepsilon(\varepsilon-\mu) \frac{\partial f}{\partial \varepsilon} \sigma_{a b}^{\mathrm{A}}(\varepsilon, T=0) \\
=-\frac{k_{\mathrm{B}}}{e} \int d \varepsilon s(\varepsilon) \frac{\partial \sigma_{a b}^{\mathrm{A}}(\varepsilon, T=0)}{\partial \varepsilon}
\end{gathered}
$$

The well-known Mott relation is obtained as $\alpha_{a b}^{\mathrm{A}} \sim$ $-\frac{\pi^{2} k_{\mathrm{B}}^{2} T}{3 e} \frac{\partial \sigma_{a b}(\mu, T=0)}{\partial \mu}$, assuming that $\sigma_{a b}^{\mathrm{A}}(\varepsilon, T=0) \propto \varepsilon-\mu$ for $\varepsilon \sim \mu$ at the low-temperature limit.

Figures 2(b) and 2(c) display the $\mu$ dependence of AHC and ANC. A shift of the chemical potential corresponds to the rigid band picture, where $\mu>0$ and $\mu<0$ represent electron and hole dopings, respectively. Note that the agreement of the ANCs calculated via Eqs. (3) and (5) is confirmed numerically as shown in Fig. 2(c). The calculated values of AHC and ANC are consistent with previous experimental and theoretical studies [6-8,23,24,51]. Slight differences with previous theoretical calculations may come from the atomic position of $\mathrm{S}$ atoms which is optimized in Refs. [6-8] whereas is adopted from the experimental value in this work.

Focusing on the ANC for $\mu \leqslant 0$, one can see that the absolute value of the ANC is large at $\mu \sim 0$ and $-0.113 \mathrm{eV}$ with opposite signs. This implies that In doping induces the sign change and/or the enhancement of the ANE as will be explicitly shown later. We also note that for qualitative understanding of the $\mu$ dependence of the ANC, the generalized Mott formula in Eq. (6) is useful. Since the entropy density $s(\varepsilon)$ is an even function with respect to $\varepsilon-\mu$ and rapidly decreases for $|\varepsilon-\mu| \gtrsim k_{\mathrm{B}} T$, one can roughly estimate the sign and magnitude of the ANC from $\frac{\partial \sigma_{a b}(\varepsilon, T=0)}{\partial \varepsilon}$ for $|\varepsilon-\mu| \lesssim k_{\mathrm{B}} T$. On one hand, around $\mu=0 \mathrm{eV}, \sigma_{a b}(\mu, T=0)$ shows a steep increase as shown in Fig. 2(b) and the resulting ANC $\alpha_{x y}^{\mathrm{A}}=-1.34 \mathrm{~A} \mathrm{~K}^{-1} \mathrm{~m}^{-1}$. On the other hand, around $\mu=-0.113 \mathrm{eV}, \sigma_{a b}(\mu, T=$ $0)$ shows a steep decrease and the resulting $\mathrm{ANC} \alpha_{x y}^{\mathrm{A}}=2.52 \mathrm{~A} \mathrm{~K}^{-1} \mathrm{~m}^{-1}$.

Next, let us move on to the In-doping dependence. The doping evolutions of the band structures and net magnetization in the presence of spin-orbit coupling are shown in Fig. 3. As mentioned before, the half-metallic state is realized in the nondoped case, whose majority spin DOS is metallic whereas minority spin DOS has a gap, neglecting the DOS with spinup/-down hybridization through spin-orbit coupling. Hence, when Sn atoms are substituted by In atoms, all holes are doped into majority spin states, whereas the number of electrons for minority spin states remains unchanged. This means that the magnetization at hole-doping $x$ is well described by $1-x$ of the magnetization per unit cell. The resulting magnetization decreases almost linearly with respect to the In content $x$, and the system becomes a nonmagnetic insulator at $x=$ 

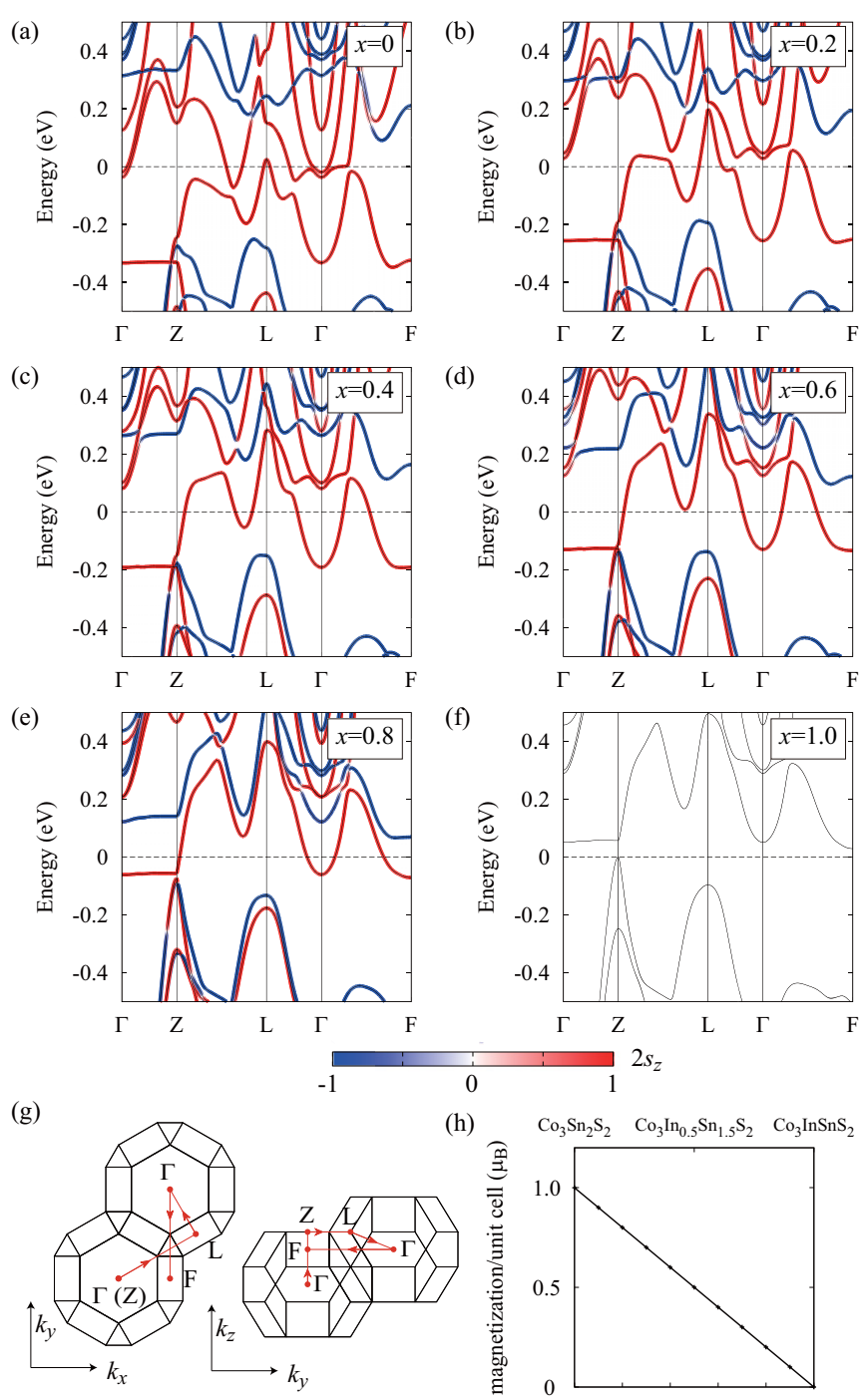

(d)

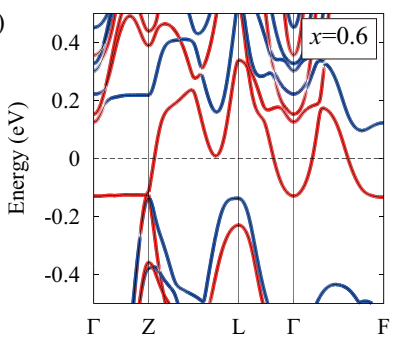

(f)

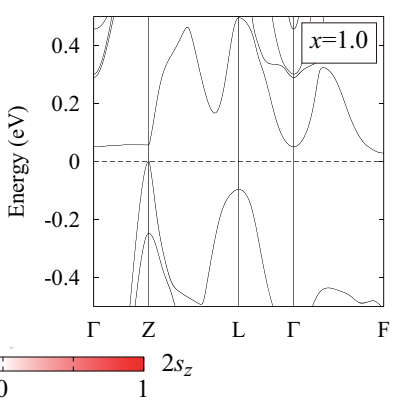

(h) $\quad \mathrm{Co}_{3} \mathrm{Sn}_{2} \mathrm{~S}_{2} \quad \mathrm{Co}_{3} \mathrm{In}_{0.5} \mathrm{Sn}_{1.5} \mathrm{~S}_{2} \quad \mathrm{Co}_{3} \mathrm{InSnS}_{2}$

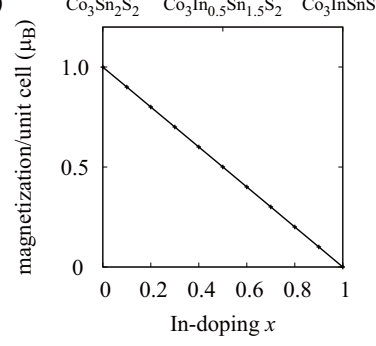

FIG. 3. (a)-(f) Band structures for $\mathrm{Co}_{3} \mathrm{In}_{x} \mathrm{Sn}_{2-x} \mathrm{~S}_{2}$ with spin-orbit coupling along high-symmetry $\boldsymbol{k}$ lines where the color map represents the spin density along the $z$ axis. (g) The $\boldsymbol{k}$ path on which the band structures are plotted in panels (a)-(f). (h) In-doping dependence of the net magnetization.

1.0 as shown in Fig. 3(h). These behaviors are qualitatively consistent with experimental results [28,29] with a slight difference of In content at which the nonmagnetic insulating phase emerges. Correspondingly, the exchange splitting due to the magnetic ordering decreases with increasing $x$, and the spin splitting of the band structure vanishes for $x=1.0$ as shown in Figs. 3(a)-3(f). The half-metallicity is retained in the whole investigated doping range of $x<1.0$. We here note that similar carrier dependence of the magnetic moment has been obtained with first-principles calculations for other half-metallic systems, such as electron-doped semiconductors $\beta-\mathrm{FeSi}_{2}$ and $\mathrm{CrSi}_{2}$ [52].

Results for the transport properties in $\mathrm{Co}_{3} \mathrm{In}_{x} \mathrm{Sn}_{2-x} \mathrm{~S}_{2}$ are summarized in Fig. 4. The AHC sensitively depends on the electronic structure, such as the details of the Fermi surfaces and the distribution of the Berry curvature in $\boldsymbol{k}$ space. As a
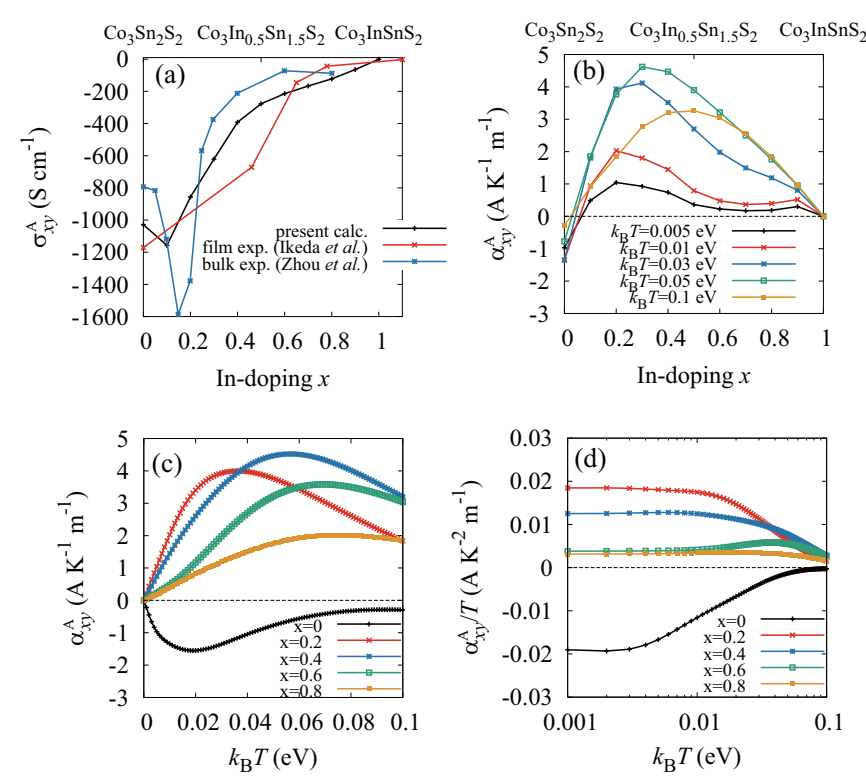

FIG. 4. (a) Doping dependence of AHC $\sigma_{x y}^{\mathrm{A}}$ at $k_{\mathrm{B}} T=0$ and (b) that of ANC $\alpha_{x y}^{\mathrm{A}}$ at finite temperatures. (c) Temperature dependence of $\alpha_{x y}^{\mathrm{A}}$ and (d) that of $\alpha_{x y}^{\mathrm{A}} / T$ at various In-doping concentrations. In panel (a), experimental data extracted from Refs. [32,45] are plotted together with calculated results for comparison.

result, the doping dependences of the AHC and ANC shown in Fig. 4 exhibit more complicated behavior than that of the magnetic moment, which linearly decreases with respect to the doping concentration as shown in Fig. 3(h) and as also demonstrated in earlier works [50,54]. Figure 4(a) shows that, for a slight hole-doped region, the AHC decreases with increasing $x$, and its absolute value reaches maximum at $x \sim 0.1$. With further increasing $x$, the AHC is suppressed and vanishes at $x=1.0$ where the system becomes nonmagnetic (see also Fig. 3). As shown in Fig. 4(b), the ANC is negative at $x=0$ and changes its sign into positive for the small doping content $x$. The absolute values of $\alpha_{x y}^{\mathrm{A}} / T$ at low temperatures are large at $x \sim 0$ and 0.2 with values of $\left|\alpha_{x y}^{\mathrm{A}}\right| / T \sim 0.02 \mathrm{~A} \mathrm{~K}^{-2} \mathrm{~m}^{-1}$. The ANC decreases with further increasing $x$, reflecting the suppression of the magnetization. These behaviors of AHC and ANC are similar to those with decreasing the chemical potential for the calculation of $x=0$ shown in Figs. 2(b) and 2(c), that is, the rigid band picture works well for the small doping region. Note that for $0.1 \lesssim x \lesssim 0.2$, experimental AHC data in the bulk show significant enhancement of the AHC [32], whereas calculated AHC is not so much enhanced. A possible origin of such behavior is a disorder effect as pointed out by several authors $[30,55,56]$, which is not considered in this work. To elucidate the enhancement of the ANC for these doping concentrations, the temperature dependence of the ANC is shown in Figs. 4(c) and 4(d). For large doping $x \geqslant 0.6, \alpha_{x y}^{\mathrm{A}}$ shows linear $T$ dependence in the wide temperature range of $k_{\mathrm{B}} T \lesssim 0.01 \mathrm{eV}$, whereas for $x=0(0.2)$, that exhibits logarithmic $T$ dependence for $0.005 \mathrm{eV} \lesssim k_{\mathrm{B}} T \lesssim$ $0.04 \mathrm{eV}\left(0.02 \mathrm{eV} \lesssim k_{\mathrm{B}} T \lesssim 0.05 \mathrm{eV}\right)$. These behaviors could be due to the stationary points in the nodal rings rather than Weyl nodes since Weyl nodes are located away from the Fermi level by $\sim 0.065 \mathrm{eV}$ in the present system. The chemical 

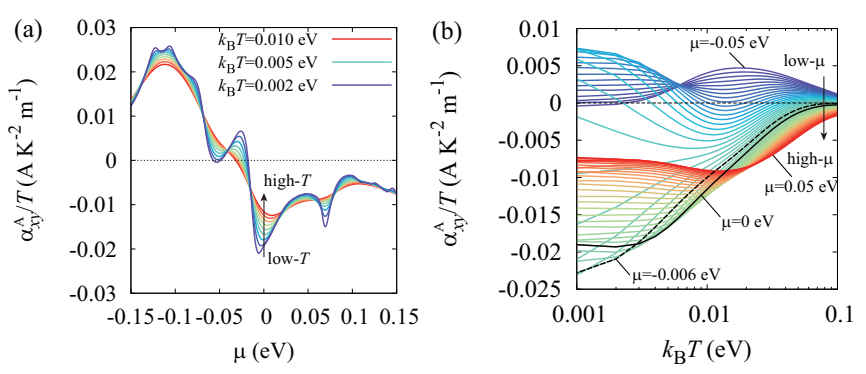

FIG. 5. (a) The chemical potential dependence of $\alpha_{x y}^{\mathrm{A}} / T$ for various values of temperature with $0.002 \mathrm{eV} \leqslant k_{\mathrm{B}} T \leqslant 0.01 \mathrm{eV}$ in $0.001 \mathrm{eV}$ steps and (b) temperature dependence of $\alpha_{x y}^{\mathrm{A}} / T$ for various values of the chemical potential with $-0.05 \mathrm{eV} \leqslant \mu \leqslant 0.05 \mathrm{eV}$ in $0.002 \mathrm{eV}$ steps at $x=0$.

potential and temperature dependences of $\alpha_{x y}^{\mathrm{A}} / T$ at $x=0$ for small $\mu$ and the $T$ region shown in Fig. 5 provide supporting evidence for this claim. We can see that for certain values of $\mu, \alpha_{x y}^{\mathrm{A}}$ shows nonlinear $T$ dependence and its temperature range highly depends on detailed values of $\mu$ from Figs. 5(a) and 5(b). For instance, for $\mu=0 \mathrm{eV}$, nonlinear $T$ dependence of $\alpha_{x y}^{\mathrm{A}}$ is observed down to $k_{\mathrm{B}} T \sim 0.005 \mathrm{eV}$ as mentioned before, whereas for $\mu=-0.006 \mathrm{eV}, \alpha_{x y}^{\mathrm{A}}$ shows nonlinear $T$ dependence down to $k_{\mathrm{B}} T \sim 0.002 \mathrm{eV}$ as shown in Fig. 5(b). Considering the energy location of Weyl nodes, which is obtained as $\sim 0.065 \mathrm{eV}$ in our calculation, Weyl nodes could not give significant contributions to $\alpha_{x y}$ for $\mu \sim 0$ at low temperatures since the Berry curvature only of the bands with the energy satisfying the relation $\left|\varepsilon_{n k}-\mu\right| \lesssim k_{\mathrm{B}} T$ contribute to the ANC according to Eq. (3). On the other hand, nodal lines extend over finite energy regions $[-0.1,0.14 \mathrm{eV}]$ across the Fermi level, and those stationary points play significant roles to enhance ANC, consistently with the previous theoretical study [24].

In order to get clear insight into the low-temperature behavior of the ANC, let us perform the detailed analysis of the topological properties of the electronic structures which are reflected in the Berry curvature $\Omega_{n, a b}(\boldsymbol{k})$. As mentioned before, the ANC is dominated by $\frac{\partial \sigma_{a b}(\varepsilon, T=0)}{\partial \varepsilon}$ for $|\varepsilon-\mu| \lesssim k_{\mathrm{B}} T$, (a)

$$
x=0
$$

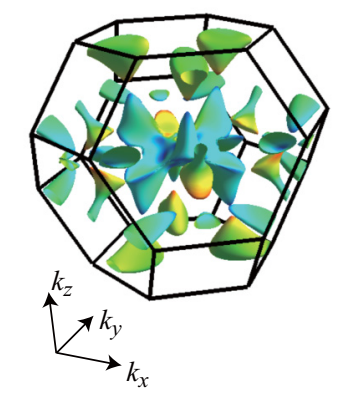

(b)

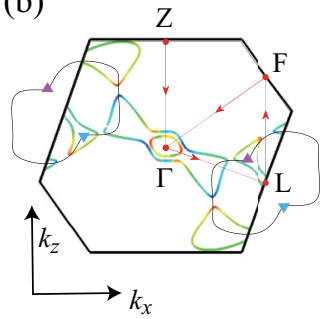

(c)

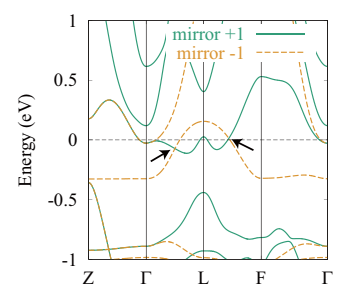

$x=0.2$

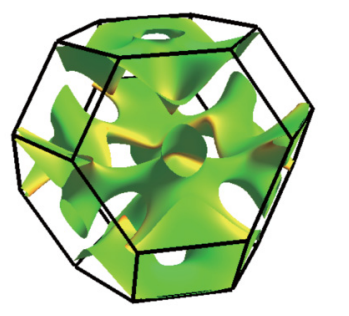

$-8$ $x=0.4$
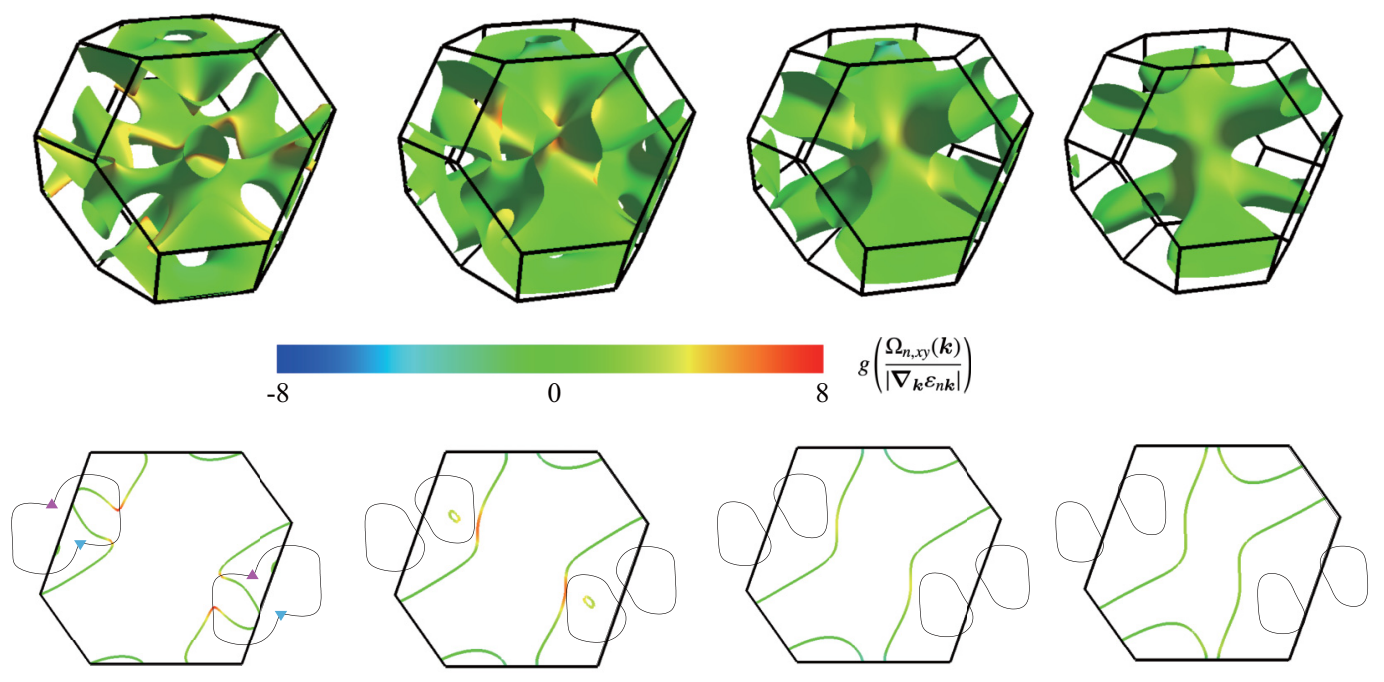

$x=0.6$

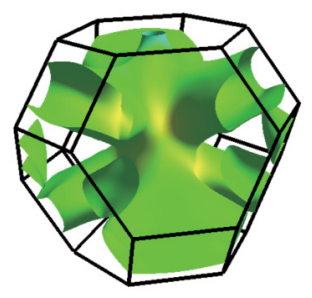

$g\left(\frac{\Omega_{n, x y}(k)}{\left|\nabla_{k} \varepsilon_{n k}\right|}\right)$

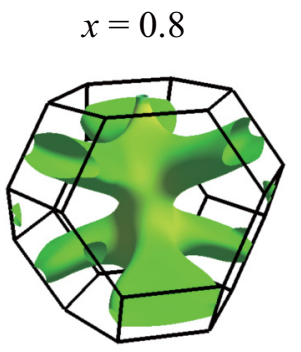

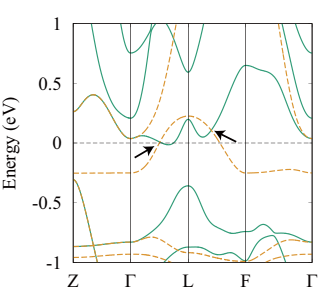
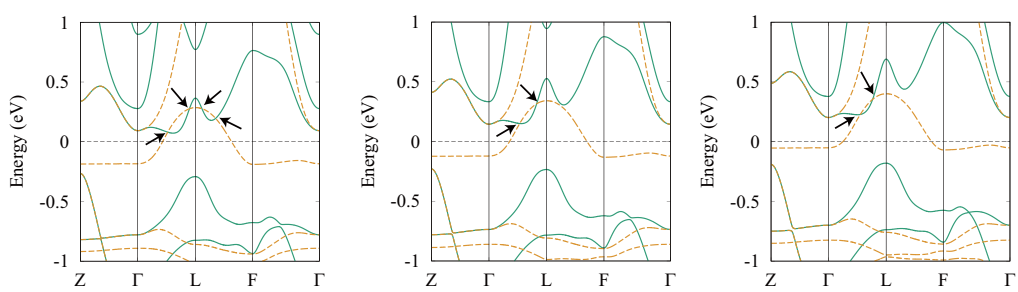

FIG. 6. (a) Fermi surfaces for $\mathrm{Co}_{3} \operatorname{In}_{x} \mathrm{Sn}_{2-x} \mathrm{~S}_{2}$ where the color map represents the intensity of $\frac{\Omega_{n, x y}(\boldsymbol{k})}{\left|\nabla_{\boldsymbol{k}} \varepsilon_{n k}\right|}$ through the logarithmic function $g(x) \equiv \operatorname{sgn}(x) \ln (1+|x|)$. (b) Those on the $k_{x}-k_{z}$ plane at $k_{y}=0$. The black solid line represents the nodal lines in the absence of spin-orbit coupling. The upper (lower) triangles on the nodal lines denote the Weyl nodes with topological charge $+1(-1)$ in the presence of spin-orbit coupling. The $\boldsymbol{k}$ path along which band structures plotted in panel (c) is shown by red arrows in the leftmost panel. (c) Majority spin band structures in the absence of the spin-orbit coupling. The solid and dashed lines represent the energy bands with eigenvalues +1 and -1 of the mirror symmetry operator on the $k_{x}-k_{z}$ plane, respectively. Band crossings corresponding to nodal lines are shown by arrows. (a) and (b) are created with the use of the FERMISURFER code [53]. 
whose explicit form is given as follows [23]:

$$
\frac{\partial \sigma_{a b}^{\mathrm{A}}(\varepsilon, T=0)}{\partial \varepsilon}=-\frac{e^{2}}{\hbar} \sum_{n} \int_{\varepsilon_{n k}=\varepsilon} \frac{d S_{\boldsymbol{k}}}{(2 \pi)^{3}} \frac{\Omega_{n, a b}(\boldsymbol{k})}{\left|\nabla_{\boldsymbol{k}} \varepsilon_{n \boldsymbol{k}}\right|},
$$

where $\int_{\varepsilon_{n k}=\varepsilon} d S_{\boldsymbol{k}}$ represents the $\boldsymbol{k}$ integration over the isoenergy surfaces for $\varepsilon_{n k}=\varepsilon$. From Eqs. (6) and (7), one can see that the qualitative behavior of the ANC at low temperatures are governed by the distribution of the $\frac{\Omega_{n, a b}(\boldsymbol{k})}{\left|\nabla_{\boldsymbol{k}} \varepsilon_{n k}\right|}$ near the Fermi surfaces. In Fig. 6(a), we show the Fermi surfaces with $\frac{\Omega_{n, a b}(\boldsymbol{k})}{\left|\nabla_{\boldsymbol{k}} \varepsilon_{n k}\right|}$. The value of $\frac{\Omega_{n, a b}(\boldsymbol{k})}{\left|\nabla_{\boldsymbol{k}} \varepsilon_{n \boldsymbol{k}}\right|}$ is positive on the small portions of the Fermi surfaces for $x=0$, whereas on the wider region, $\frac{\Omega_{n, a b}(\boldsymbol{k})}{\left|\nabla_{\boldsymbol{k}} \varepsilon_{n \boldsymbol{k}}\right|}$ becomes negative. As a result, the $\boldsymbol{k}$ integration over the Fermi surfaces of $\frac{\Omega_{n, a b}(\boldsymbol{k})}{\left|\nabla_{k} \varepsilon_{n k}\right|}$ is negative, resulting in $\alpha_{x y}^{\mathrm{A}}<0$ for $\mathrm{Co}_{3} \mathrm{Sn}_{2} \mathrm{~S}_{2}$ as shown in Fig. 4(b). On the other hand, for $x=0.2$, the area of the Fermi surfaces on which $\frac{\Omega_{n, a b}(\boldsymbol{k})}{\left|\nabla_{\boldsymbol{k}} \varepsilon_{n k}\right|}$ is negative becomes small, and the resulting ANC is positive. The absolute value of $\frac{\Omega_{n, a b}(\boldsymbol{k})}{\left|\nabla_{\boldsymbol{k}} \varepsilon_{n k}\right|}$ decreases with further increasing $x$, and the ANCs for large $x$ have small values [see also Fig. 4(b)].

We here demonstrate that the intensity of the Berry curvature on Fermi surfaces is closely related to the nodal lines. As shown in Fig. 6(b), the nodal lines appear on the mirror symmetry planes in the absence of the spin-orbit coupling. The spin-orbit coupling gaps out the nodal lines but leaves the degeneracy of the energy at $\sim 0.065 \mathrm{eV}$, resulting in the Weyl nodes for $x=0[6,7]$. In this case, the energy bands characterized by different eigenvalues \pm 1 of the mirror symmetry operator produce nodal lines due to its crossing [see majority spin band structures in the absence of spin-orbit coupling shown in Fig. 6(c)]. This origin of nodal lines is similar to those in archetypal nonmagnetic nodal line semimetals $\mathrm{Ca}_{3} \mathrm{P}_{2}$ [57,58] and $\mathrm{CaAg} X$ ( $X=\mathrm{P}, \mathrm{As})$ [59].

The hole doping decreases the Fermi energy and as a result shifts the Weyl nodes away from the Fermi level. The nodal rings are located around the Fermi energy for small hole doping, and for $x \lesssim 0.2$, the nodal lines surrounding the $L$ point cross the Fermi surfaces as shown in Fig. 6(b). The band at the $L$ point near the Fermi energy with mirror eigenvalue +1 shifts upward in energy with increasing $x$ and crosses with the band having mirror eigenvalue -1 for $x \sim 0.3$ as shown in Fig. 6(c). Then, the nodal lines are split into two rings as shown in Fig. 6(b) with the annihilation of Weyl nodes in the presence of the spin-orbit coupling. The nodal lines still cross the Fermi surfaces for $x<0.4$ and give the significant contribution to the ANC. For $x \gtrsim 0.6$, the nodal lines are located far from the Fermi level, resulting in the small Berry curvature on the whole Fermi surfaces. One can clearly see that the intensity of the Berry curvature is large near the nodal lines.

\section{SUMMARY AND DISCUSSIONS}

In this work, we investigated the magnetic and transport properties in $\mathrm{Co}_{3} \mathrm{In}_{x} \mathrm{Sn}_{2-x} \mathrm{~S}_{2}$ based on first-principles calculations in which the In-doping effect is treated within the virtual crystal approximation. We show that the anomalous Hall and Nernst conductivities show complicated behaviors with varying $x$ against linearly reduced magnetization with respect to In content $x$ due to the half-metallic electronic states. The Nernst conductivity has large values for $x \sim 0$ and 0.2 with opposite signs and shows logarithmic temperature dependence consistently with the previous theoretical study [24]. We also clarify that the low-temperature behavior of the anomalous Nernst conductivity can be understood by the distribution of the Berry curvature divided by the Fermi velocity. The close relationships among the Berry curvature, nodal rings, and anomalous Nernst effect are explicitly demonstrated. The intensity of the Berry curvature has a large value near the nodal rings for small hole doping, leading to a large anomalous Nernst conductivity. The In doping induces the reconstruction of the nodal rings and moves the nodal lines far away from the Fermi level, resulting in the small Berry curvature on the Fermi surfaces. Our results give a qualitative understanding of the thermoelectric transport in $\mathrm{Co}_{3} \mathrm{In}_{x} \mathrm{Sn}_{2-x} \mathrm{~S}_{2}$ and encourage experimental measurements of the anomalous Nernst effect in $\mathrm{Co}_{3} \mathrm{In}_{x} \mathrm{Sn}_{2-x} \mathrm{~S}_{2}$.

Lastly, we comment on the important issues which are not addressed in this paper. In the present paper, we have focused on the intrinsic contribution to the anomalous transports and neglected the extrinsic effects, such as side jump and skew scattering [1]. Effects of the structural disorder, however, is inevitable in doped compounds, in general, and might give considerably large extrinsic contribution to the thermoelectric transport pointed by several authors [30,55,56]. A quantitative study on extrinsic contributions using first-principles calculations is a remaining issue in a future work.

\section{ACKNOWLEDGMENTS}

This research was supported by JSPS KAKENHI Grants No. JP15H05883 (J-Physics), No. JP18H04230, No. JP19H01842, No. JP20H01830, No. JP20H05262, No. JP20K05299, No. JP20K21067, No. JP21H01031, No. JP21H01789, and No. JP21H04437. We also acknowledge support from JST PRESTO Grant No. JPMJPR17N8 and JST CREST Grant No. JPMJCR18T2. A part of the numerical calculations was carried out using MASAMUNE-IMR of the Center for Computational Materials Science, Institute for Materials Research, Tohoku University. Y.Y. thanks H. Kusunose for sharing computer facilities.
[1] N. Nagaosa, J. Sinova, S. Onoda, A. H. MacDonald, and N. P. Ong, Rev. Mod. Phys. 82, 1539 (2010).

[2] D. Xiao, M.-C. Chang, and Q. Niu, Rev. Mod. Phys. 82, 1959 (2010).
[3] N. P. Armitage, E. J. Mele, and A. Vishwanath, Rev. Mod. Phys. 90, 015001 (2018).

[4] Y. Tokura, K. Yasuda, and A. Tsukazaki, Nat. Rev. Phys. 1, 126 (2019). 
[5] C. Fu, Y. Sun, and C. Felser, APL Mater. 8, 040913 (2020).

[6] E. Liu, Y. Sun, N. Kumar, L. Muechler, A. Sun, L. Jiao, S.-Y. Yang, D. Liu, A. Liang, Q. Xu, J. Kroder, V. Süß, H. Borrmann, C. Shekhar, Z. Wang, C. Xi, W. Wang, W. Schnelle, S. Wirth, Y. Chen, S. T. B. Goennenwein, and C. Felser, Nat. Phys. 14, 1125 (2018).

[7] Q. Wang, Y. Xu, R. Lou, Z. Liu, M. Li, Y. Huang, D. Shen, H. Weng, S. Wang, and H. Lei, Nat. Commun. 9, 3681 (2018).

[8] S. N. Guin, P. Vir, Y. Zhang, N. Kumar, S. J. Watzman, C. Fu, E. Liu, K. Manna, W. Schnelle, J. Gooth, C. Shekhar, Y. Sun, and C. Felser, Adv. Mater. 31, 1806622 (2019).

[9] A. Sakai, Y. P. Mizuta, A. A. Nugroho, R. Sihombing, T. Koretsune, M.-T. Suzuki, N. Takemori, R. Ishii, D. NishioHamane, R. Arita, P. Goswami, and S. Nakatsuji, Nat. Phys. 14, 1119 (2018).

[10] S. N. Guin, K. Manna, J. Noky, S. J. Watzman, C. Fu, N. Kumar, W. Schnelle, C. Shekhar, Y. Sun, J. Gooth, and C. Felser, NPG Asia Mater. 11, 16 (2019).

[11] R. Weihrich and I. Anusca, Z. Anorg. Allg. Chem. 632, 1531 (2006).

[12] M. Holder, Y. S. Dedkov, A. Kade, H. Rosner, W. Schnelle, A. Leithe-Jasper, R. Weihrich, and S. L. Molodtsov, Phys. Rev. B 79, 205116 (2009).

[13] W. Schnelle, A. Leithe-Jasper, H. Rosner, F. M. Schappacher, R. Pöttgen, F. Pielnhofer, and R. Weihrich, Phys. Rev. B 88, 144404 (2013).

[14] K. Fujiwara, J. Ikeda, J. Shiogai, T. Seki, K. Takanashi, and A. Tsukazaki, Jpn. J. Appl. Phys. 58, 050912 (2019).

[15] J. Ikeda, K. Fujiwara, J. Shiogai, T. Seki, K. Nomura, K. Takanashi, and A. Tsukazaki, Commun. Mater. 2, 18 (2021).

[16] D. F. Liu, A. J. Liang, E. K. Liu, Q. N. Xu, Y. W. Li, C. Chen, D. Pei, W. J. Shi, S. K. Mo, P. Dudin, T. Kim, C. Cacho, G. Li, Y. Sun, L. X. Yang, Z. K. Liu, S. S. P. Parkin, C. Felser, and Y. L. Chen, Science 365, 1282 (2019).

[17] L. Jiao, Q. Xu, Y. Cheon, Y. Sun, C. Felser, E. Liu, and S. Wirth, Phys. Rev. B 99, 245158 (2019).

[18] N. Morali, R. Batabyal, P. K. Nag, E. Liu, Q. Xu, Y. Sun, B. Yan, C. Felser, N. Avraham, and H. Beidenkopf, Science 365, 1286 (2019).

[19] J.-X. Yin, S. S. Zhang, G. Chang, Q. Wang, S. S. Tsirkin, Z. Guguchia, B. Lian, H. Zhou, K. Jiang, I. Belopolski, N. Shumiya, D. Multer, M. Litskevich, T. A. Cochran, H. Lin, Z. Wang, T. Neupert, S. Jia, H. Lei, and M. Z. Hasan, Nat. Phys. 15, 443 (2019).

[20] J.-X. Yin, N. Shumiya, Y. Jiang, H. Zhou, G. Macam, H. O. M. Sura, S. S. Zhang, Z.-J. Cheng, Z. Guguchia, Y. Li, Q. Wang, M. Litskevich, I. Belopolski, X. P. Yang, T. A. Cochran, G. Chang, Q. Zhang, Z.-Q. Huang, F.-C. Chuang, H. Lin, H. Lei, B. M. Andersen, Z. Wang, S. Jia, and M. Z. Hasan, Nat. Commun. 11, 4415 (2020).

[21] H. Yang, W. You, J. Wang, J. Huang, C. Xi, X. Xu, C. Cao, M. Tian, Z.-A. Xu, J. Dai, and Y. Li, Phys. Rev. Mater. 4, 024202 (2020).

[22] A. A. Burkov, Phys. Rev. Lett. 113, 187202 (2014).

[23] M. P. Ghimire, J. I. Facio, J.-S. You, L. Ye, J. G. Checkelsky, S. Fang, E. Kaxiras, M. Richter, and J. van den Brink, Phys. Rev. Res. 1, 032044(R) (2019).

[24] S. Minami, F. Ishii, M. Hirayama, T. Nomoto, T. Koretsune, and R. Arita, Phys. Rev. B 102, 205128 (2020).
[25] X. Chen, M. Wang, C. Gu, S. Wang, Y. Zhou, C. An, Y. Zhou, B. Zhang, C. Chen, Y. Yuan, M. Qi, L. Zhang, H. Zhou, J. Zhou, Y. Yao, and Z. Yang, Phys. Rev. B 100, 165145 (2019).

[26] Z. Y. Liu, T. Zhang, S. X. Xu, P. T. Yang, Q. Wang, H. C. Lei, Y. Sui, Y. Uwatoko, B. S. Wang, H. M. Weng, J. P. Sun, and J.-G. Cheng, Phys. Rev. Mater. 4, 044203 (2020).

[27] Z. Guguchia, J. A. T. Verezhak, D. J. Gawryluk, S. S. Tsirkin, J. X. Yin, I. Belopolski, H. Zhou, G. Simutis, S. S. Zhang, T. A. Cochran, G. Chang, E. Pomjakushina, L. Keller, Z. Skrzeczkowska, Q. Wang, H. C. Lei, R. Khasanov, A. Amato, S. Jia, T. Neupert, H. Luetkens, and M. Z. Hasan, Nat. Commun. 11, 559 (2020).

[28] M. A. Kassem, Y. Tabata, T. Waki, and H. Nakamura, J. Phys. Soc. Jpn. 85, 064706 (2016).

[29] J. Corps, P. Vaqueiro, A. Aziz, R. Grau-Crespo, W. Kockelmann, J.-C. Jumas, and A. V. Powell, Chem. Mater. 27, 3946 (2015).

[30] J. Shen, Q. Zeng, S. Zhang, H. Sun, Q. Yao, X. Xi, W. Wang, G. Wu, B. Shen, Q. Liu, and E. Liu, Adv. Funct. Mater. 30, 2000830 (2020).

[31] G. S. Thakur, P. Vir, S. N. Guin, C. Shekhar, R. Weihrich, Y. Sun, N. Kumar, and C. Felser, Chem. Mater. 32, 1612 (2020).

[32] H. Zhou, G. Chang, G. Wang, X. Gui, X. Xu, J.-X. Yin, Z. Guguchia, S. S. Zhang, T.-R. Chang, H. Lin, W. Xie, M. Z. Hasan, and S. Jia, Phys. Rev. B 101, 125121 (2020).

[33] J. Rothballer, F. Bachhuber, S. M. Rommel, T. Söhnel, and R. Weihrich, RSC Adv. 4, 42183 (2014).

[34] K. Momma and F. Izumi, J. Appl. Crystallogr. 44, 1272 (2011).

[35] P. Blaha, K. Schwarz, G. Madsen, D. Kvasnicka, and J. Luitz, An Augmented Plane Wave + Local Orbitals Program for Calculating Crystal Properties (Technische Universität Wien, Vienna, 2001).

[36] P. Blaha, K. Schwarz, F. Tran, R. Laskowski, G. K. H. Madsen, and L. D. Marks, J. Chem. Phys. 152, 074101 (2020).

[37] J. P. Perdew, K. Burke, and M. Ernzerhof, Phys. Rev. Lett. 77, 3865 (1996).

[38] Y. Fu and D. J. Singh, Phys. Rev. B 100, 045126 (2019).

[39] N. Marzari, A. A. Mostofi, J. R. Yates, I. Souza, and D. Vanderbilt, Rev. Mod. Phys. 84, 1419 (2012).

[40] G. Pizzi, V. Vitale, R. Arita, S. Blügel, F. Freimuth, G. Géranton, M. Gibertini, D. Gresch, C. Johnson, T. Koretsune, J. Ibañez-Azpiroz, H. Lee, J.-M. Lihm, D. Marchand, A. Marrazzo, Y. Mokrousov, J. I. Mustafa, Y. Nohara, Y. Nomura, L. Paulatto, S. Poncé, T. Ponweiser, J. Qiao, F. Thöle, S. S. Tsirkin, M. Wierzbowska, N. Marzari, D. Vanderbilt, I. Souza, A. A. Mostofi, and J. R. Yates, J. Phys.: Condens. Matter 32, 165902 (2020).

[41] J. Kuneš, R. Arita, P. Wissgott, A. Toschi, H. Ikeda, and K. Held, Comput. Phys. Commun. 181, 1888 (2010).

[42] X. Wang, J. R. Yates, I. Souza, and D. Vanderbilt, Phys. Rev. B 74, 195118 (2006).

[43] F. D. M. Haldane, Phys. Rev. Lett. 93, 206602 (2004).

[44] D. Xiao, Y. Yao, Z. Fang, and Q. Niu, Phys. Rev. Lett. 97, 026603 (2006).

[45] The $\mathrm{Co}_{3} \mathrm{In}_{x} \mathrm{Sn}_{2-x} \mathrm{~S}_{2}$ films were grown on $\mathrm{Al}_{2} \mathrm{O}_{3}$ (0001) substrates by cosputtering [14]. The $c$-axis oriented growth was confirmed by x-ray diffraction measurements. The thicknesses of the films were approximately $40 \mathrm{~nm}$. After cooling the samples in an out-of-plane magnetic field of $1 \mathrm{~T}$, the electrical transport properties were measured at $2 \mathrm{~K} . \sigma_{x y}$ was calculated 
by $\sigma_{x y}=\rho_{y x} /\left(\rho_{x x}^{2}+\rho_{y x}^{2}\right)$, where $\rho_{x x}$ is electrical resistivity and $\rho_{y x}$ is Hall resistivity. The detailed transport properties will be reported elsewhere.

[46] R. R. Birss, Symmetry and Magnetism (North-Holland, Amsterdam, 1964).

[47] M. Seemann, D. Ködderitzsch, S. Wimmer, and H. Ebert, Phys. Rev. B 92, 155138 (2015).

[48] H. Watanabe and Y. Yanase, Phys. Rev. B 98, 245129 (2018).

[49] S. Hayami, M. Yatsushiro, Y. Yanagi, and H. Kusunose, Phys. Rev. B 98, 165110 (2018).

[50] M.-T. Suzuki, T. Koretsune, M. Ochi, and R. Arita, Phys. Rev. B 95, 094406 (2017).

[51] A. Ozawa and K. Nomura, J. Phys. Soc. Jpn. 88, 123703 (2019).

[52] D. J. Singh and D. Parker, Sci. Rep. 3, 3517 (2013).
[53] M. Kawamura, Comput. Phys. Commun. 239, 197 (2019).

[54] M. Naka, S. Hayami, H. Kusunose, Y. Yanagi, Y. Motome, and H. Seo, Phys. Rev. B 102, 075112 (2020).

[55] L. Ding, J. Koo, L. Xu, X. Li, X. Lu, L. Zhao, Q. Wang, Q. Yin, H. Lei, B. Yan, Z. Zhu, and K. Behnia, Phys. Rev. X 9, 041061 (2019).

[56] M. Papaj and L. Fu, Phys. Rev. B 103, 075424 (2021).

[57] L. S. Xie, L. M. Schoop, E. M. Seibel, Q. D. Gibson, W. Xie, and R. J. Cava, APL Mater. 3, 083602 (2015).

[58] Y.-H. Chan, C.-K. Chiu, M. Y. Chou, and A. P. Schnyder, Phys. Rev. B 93, 205132 (2016).

[59] A. Yamakage, Y. Yamakawa, Y. Tanaka, and Y. Okamoto, J. Phys. Soc. Jpn. 85, 013708 (2016). 